CORRESPONDENCE

\title{
Arterial thromboembolism in multiple myeloma in the context of modern anti-myeloma therapy
}

(c) The Author(s) 2021

Blood Cancer Journal (2021)11:121; https://doi.org/

10.1038/s41408-021-00513-4

\section{Dear Editor}

Although the incidence and risk factors of venous thromboembolism [VTE] is well characterized in multiple myeloma [MM], little is known regarding the characterization of arterial thromboembolism [ATE] in the context of modern anti-myeloma therapy. Since MM is a cancer of older adults with several shared risk factors for cancer and cardiovascular disease, ATE remains an area of major concern. Notably, population-based studies from Sweden have demonstrated an increased risk of ATE in MM compared to matched controls, with an incidence of $3.8 \%$ in the first year [1]. Another study by the HOVON Group reported a high ATE incidence of $5.6 \%$ among newly diagnosed transplant-eligible MM patients receiving doxorubicin-based regimens [2]. However, data from older literature captured ATE incidence prior to the introduction of proteasome inhibitors and immunomodulatory drugs (IMiDs). The objective of our retrospective cohort study was to investigate the incidence, risk factors, and nature of ATE events in newly diagnosed $\mathrm{MM}$ in the context of modern anti-myeloma therapy.

We included all consecutive $\mathrm{MM}$ patients treated at the Cleveland Clinic from 1/1/2008 to 12/31/2018. We identified potential baseline disease-related, patient-related and treatmentrelated risk factors a priori and extracted from medical records. The primary endpoint was to estimate ATE incidence in the first year after treatment initiation. Secondary endpoints were to identify risk factors for ATE and association of ATE with overall survival [OS].

We calculated the cumulative incidence of ATE by 12 months. Death without ATE was a competing risk for ATE. Fine and Gray regression was used to identify univariate risk factors for ATE and results were reported as hazard ratio [HR] with $95 \%$ confidence intervals $[\mathrm{Cl}]$. We tested the following pre-treatment variables: year of treatment, age, sex, race, immunoglobulin subtype, International Staging System [ISS] stage, bone marrow plasma cell percentage, M-protein, involved/uninvolved free light chain ratio, karyotype, high-risk FISH, lactate dehydrogenase, creatinine, calcium, hemoglobin, prior ATE, prior VTE, body mass index, diabetes mellitus, chronic kidney disease, hypertension, hyperlipidemia, total leukocyte count, platelet count, liver disease, acute infection (within 90 days before treatment), erythropoietin use, clotting disorders, autoimmune disease, hyperviscosity, dexamethasone dose, doxorubicin use, multiagent chemotherapy, smoking status, IMiD use, and concurrent anti-platelet/anti-thrombotic therapy. If $\mathrm{HR}$ could not be calculated, Gray test was used instead to assess association with ATE; this occurs for variables with categories that have $0 \%$ or $100 \%$ ATE events. Survival based on occurrence of ATE was assessed by landmark analyses at 6 and 12 months. For this analysis, Kaplan-Meier survival estimates at 5 years were calculated for those with or without ATE by the landmark along with log-rank test $p$ value.

A total of 1029 consecutive patients met inclusion criteria, of whom 934 patients with available data on initial anti-myeloma therapy were analyzed. The baseline clinical and demographic characteristics are summarized in Table 1 . The median age at treatment initiation was 63 years [range, 22-94]. Approximately one-fifth of patients were Black and more than half were male. The cohort was roughly equally divided into ISS stages I, II, and III disease, with one-fourth of patients having high-risk FISH cytogenetics at diagnosis. ATE before MM diagnosis was present in $12 \%$ of patients. Approximately one-fifth were actively smoking or had quit within 10 years prior to MM diagnosis. A 3-drug induction regimen with VRD (bortezomiblenalidomide-dexamethasone) or VCD (bortezomib-cyclophosphamide-dexamethasone) was initiated in $52 \%$ of patients. Less than $6 \%$ had received high-dose dexamethasone [>16o mg per cycle]. The following anti-platelet/anti-thrombotic agents were administered: none [33\%], low-dose aspirin [55\%], prophylactic low molecular weight heparin [LMWH] (4\%), and warfarin or therapeutic LMWH [7\%].

A total of 25 ATE events were observed within a year of treatment initiation. The cumulative incidence of ATE at 6 and 12 months was $2.0 \%$ [95\% Cl, 1.2-3.0] and $2.7 \%$ [95\% Cl, 1.8-4.0] respectively. The Kaplan-Meier curve for cumulative incidence of ATE, along with that of VTE for comparison, is shown in Fig. 1. The median time to ATE from treatment initiation was 4.8 months [range, $0.2-11.5 \mathrm{mo}$.]. The nature of ATEs are as follows: acute ischemic stroke $[n=10 ; 40 \%]$, acute myocardial infarction $[n=10$; $40 \%]$, transient ischemic attack $[n=2 ; 8 \%]$, peripheral artery embolism $[n=2 ; 8 \%]$, and superior mesenteric artery thrombosis [ $n=1 ; 4 \%]$. Of 15 patients for whom response status at the time of ATE was available, 4 had very good partial response [VGPR], 7 had $\mathrm{PR}$, and 4 had stable disease.

On univariate analysis, the following factors were significantly predictive of ATE: ISS stage III disease [vs I/II; HR $=2.62 ; 95 \% \mathrm{Cl}$, $1.06-6.51 ; p=0.038]$, prior ATE $[\mathrm{HR}=6.86 ; 95 \% \mathrm{Cl}, 3.14-15.0 ; p<$ 0.001 ], diabetes mellitus [HR $=2.89 ; 95 \% \mathrm{Cl}, 1.28-6.52 ; p=0.011]$, chronic kidney disease [HR $=3.58 ; 95 \% \mathrm{Cl}, 1.48-8.69 ; p=0.005]$, hyperlipidemia $[\mathrm{HR}=2.66 ; 95 \% \mathrm{Cl}, 1.19-5.92 ; p=0.017]$, acute infection $[\mathrm{HR}=4.92 ; 95 \% \mathrm{Cl}, 1.71-14.2 ; p=0.003]$, and current smoker [vs former/never smoker; $\mathrm{HR}=2.37 ; 95 \% \mathrm{Cl}, 1.05-5.35$; $p=0.037]$. Of note, use of IMiDs in induction therapy was not associated with an increase in ATE risk $[\mathrm{HR}=0.54 ; 95 \% \mathrm{Cl}$, $0.24-1.20 ; p=0.13$. We did not find any significant association 
Table 1. Baseline clinical and demographic characteristics.

\section{Variable [Number of Patients with Available Data]}

Male sex [934]

Race [925]:

White

Black

Other

Age at treatment initiation[934]

Multiple Myeloma Subtype [934]:

$\lg G$
$\lg A$
$\lg M$

Others

Albumin [g/dl] [817]

B-2 microglobulin [mcg/ml] [762]

ISS stage [808]

II

Percentage BMPCs [866]

Serum M-protein [g/dl] [822]

Involved/Uninvolved sFLC ratio [743]

Abnormal Metaphase Cytogenetics [781]

High-Risk FISH Cytogenetics $^{\mathrm{a}}$ [604]

LDH > ULN [643]

History of ATE [934]

History of VTE [931]

BMI [799]

$<25$

25-29.9

$30-34.9$

$35-39.9$

$\geq 40$

History of Cardiac Disease ${ }^{\mathrm{b}}$ [933]

History of DM [934]

History of CKD [931]

History of HTN [932]

History of HLD [923]

Acute Infection at Diagnosis ${ }^{c}$ [932]

History of Autoimmune Disease [925]

Smoking History [922] ${ }^{\mathrm{d}}$

Never

Former

Current

Initial Treatment Regimen [934]:

VRD

VD

$\mathrm{RD}$

VCD

\begin{tabular}{lc}
\hline Patients [934] & \\
\hline $\boldsymbol{N}$ & $\%$ \\
\hline 516 & 55.2 \\
\hline 738 & \\
\hline 175 & 79.8 \\
\hline 12 & 18.9 \\
\hline Median: 63 years & NA \\
[22-94] & 1.3 \\
\hline
\end{tabular}

477

51.1

$209 \quad 22.4$

$11 \quad 1.2$

237

25.4

Median: $3.7 \quad$ NA

[1.1-5.2]

Median: 3.8

[0.2-78.0]

\begin{tabular}{lc}
267 & 33.0 \\
\hline 260 & 32.2 \\
\hline 281 & 34.8 \\
\hline 50 [1-100] & NA \\
\hline $\begin{array}{l}\text { Median: } 1.95 \\
\text { [0-10.49] }\end{array}$ & NA \\
\hline $80[0.5-64,775]$ & NA \\
\hline 146 & 18.7 \\
\hline 143 & 23.7 \\
\hline 178 & 27.7 \\
\hline 114 & 12.2 \\
60 & 6.4 \\
\hline
\end{tabular}

$60 \quad 6.4$

$208 \quad 26$

$291 \quad 36.4$

$195 \quad 24.4$

$66 \quad 8.3$

$39 \quad 4.9$

$164 \quad 17.6$

$157 \quad 16.8$

$106 \quad 11.4$

$464 \quad 49.8$

$288 \quad 31.2$

$37 \quad 4.0$

$60 \quad 6.5$

$523 \quad 56.7$

$222 \quad 24.1$

$177 \quad 19.2$

$378 \quad 40.5$

$204 \quad 21.8$

$179 \quad 19.2$

$104 \quad 11.1$

Table 1 continued

Variable [Number of Patients with Available Data]

Others

Patients [934]

\section{N}

69

$\%$

Dexamethasone Dose per Cycle [900]:

$\begin{array}{llr}<120 \mathrm{mg} & 168 & 18.7 \\ 120-60 \mathrm{mg} & 681 & 75.7 \\ >160 \mathrm{mg} & 51 & 5.7\end{array}$

Initial Thromboprophylaxis Regimen [863]

\begin{tabular}{llr} 
None & 288 & 33.4 \\
\hline ASA & 477 & 55.3 \\
\hline Prophylactic LMWH & 35 & 4.1 \\
\hline Warfarin/Therapeutic LMWH & 63 & 7.3
\end{tabular}

ISS International Staging System, BMPC Bone Marrow Plasma Cells, NA Not Applicable, sFLC Serum Free Light Chain, FISH Fluorescence in situ Hybridization, LDH Lactate Dehydrogenase, ULN Upper Limit of Normal, ATE Arterial Thromboembolism, VTE Venous Thromboembolism, BMI Body Mass Index, DM Diabetes Mellitus, CKD Chronic Kidney Disease, HTN Hypertension, HLD Hyperlipidemia, VRD Bortezomib-Lenalidomide-Dexamethasone, VD Bortezomib-Dexamethasone, RD Lenalidomide-Dexamethasone, VCD Bortezomib-Cyclophosphamide-Dexamethasone, ASA Aspirin, LMWH Low Molecular Weight Heparin.

${ }^{a} \mathrm{High}$-risk FISH abnormality was defined by the presence of deletion(17p), $t$ $(4 ; 14), \mathrm{t}(14 ; 16)$, and/or $\mathrm{t}(14 ; 20)$.

${ }^{\mathrm{b}} \mathrm{Cardiac}$ disease was defined as congestive heart failure, coronary artery disease [including acute myocardial infarction], and/or arrhythmia.

'Defined as acute infection within 90 days prior to treatment initiation.

${ }^{d}$ Current smokers were defined as patients who were smoking at diagnosis or had quit smoking less than 10 years before diagnosis. Former smokers were defined as patients who had quit smoking more than 10 years before diagnosis.

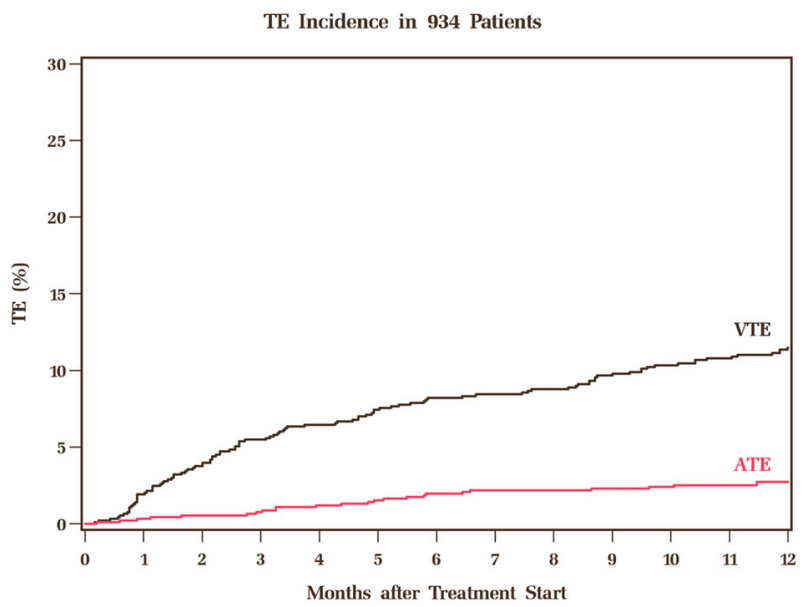

Fig. 1 Incidence of arterial and venous thromboembolism in the first year after treatment initiation. ATE Arterial Thromboembolism, VTE Venous Thromboembolism.

between the use of thromboprophylactic agents and incidence of ATE.

Subsequently, we evaluated the association of ATE with OS. Of 25 patients with ATE events, nine were alive with a median follow-up after ATE diagnosis of 23.9 months [range, 7.971.8 months]. The 5-year OS among patients with or without ATE at 6-month landmark was $39 \%$ and $60 \%$ respectively 
[ $p=0.004]$ and that at 12-month landmark was $45 \%$ and $63 \%$ respectively $[p<0.001]$. The Kaplan-Meier curves for survival using landmark analysis at 6 and 12 months is shown in the figure in Supplementary Appendix A.

Our study demonstrates that the incidence of ATE in the first year after myeloma diagnosis is $2.7 \%$ in the current era, which is substantially lower than prior estimates with cytotoxic therapies. Notably, the high ATE incidence $(5.6 \%)$ seen in the study by the HOVON group was in the context of doxorubicin-based regimens [2], which could be due to the pro-coagulant effect of doxorubicin on platelets [3]. Our data is in line with the recently published Myeloma XI trial, which showed an ATE incidence of $1.3 \%$ and $2.4 \%$ in transplant-eligible and transplant-ineligible pathways respectively [4]. Furthermore, data from our study confirms the association of ATE with worsened OS in the current era, consistent with the Swedish myeloma database and Myeloma XI trial [4,5,]. However, we acknowledge, that there may not be a causal relationship between ATE and survival, since ATE is associated with several co-morbidities and a high tumor burden, which may be the drivers of mortality in these patients. The only tumorspecific risk factor that we could identify was ISS stage III, which implies a high tumor burden. Although IMiD use is a wellestablished risk factor for VTE in MM [6], the association with ATE is less well defined. Due to a low event rate, it remains difficult to observe meaningful difference in ATE incidence between treatment arms in randomized clinical trials [7]. With a large sample size, our study shows that IMiD use may not associated with increased ATE incidence in the first year of treatment. However, prolonged IMiD maintenance therapy beyond first year may be associated with a small increase in ATE risk, as shown in the Myeloma XI trial [4].

Our study has limitations. First, our database did not have incidence of clonal hematopoiesis of indeterminate potential [CHIP], which is a non-modifiable risk-factor for atherosclerotic cardiovascular disease [8]. However, a recent study on 629 transplanted myeloma patients did not demonstrate CHIP as a predictor of ATE in this population at a median follow-up of $\sim 10$ years [9]. Second, despite a large sample size, the total number of ATE events was low, hence, multivariable analysis of risk factors could not be performed. Unfortunately, confounding can be present in retrospective analyses; hence, we need studies with more events to identify independent risk factors for ATE. Third, we did not have a matched control group of patients without myeloma. However, based on data from the SEER-Medicare linked database, the 6-month cumulative incidence of ATE in patients with cancer and matched controls is $4.7 \%$ and $2.2 \%$ respectively, with the highest risk in patients with lung cancer (8.3\%) [10]. Hence, the incidence of ATE in patients with MM treated in the modern era was comparable to Medicare enrollees without a cancer diagnosis.

In summary, we show a small but significant risk of ATE in the first year after myeloma diagnosis in the current era, with several modifiable risk factors, including diabetes, hyperlipidemia, and smoking. A history of ATE was present in $12 \%$ of patients prior to myeloma diagnosis, which was the strongest risk factor for subsequent ATE. Patients with myeloma should undergo a thorough risk assessment for ATE at diagnosis. There is a paucity of data on primary prophylaxis for ATE in patients with cancer. A phase III RCT comparing LMWH (nadroparin) to placebo for prevention of VTE and ATE in solid tumor patients demonstrated a decrease in ATE risk (stroke and peripheral thrombosis) by $50 \%$ ( $0.4 \%$ in nadroparin arm and $0.8 \%$ in placebo arm) [11]. However, the primary endpoint included a composite of VTE and ATE and lacked power to detect a difference in ATE specifically. Similarly, a recent update from the CASSINI trial demonstrated a numerically lower incidence of ATE with rivaroxaban compared to placebo (1.0\% vs $1.7 \%)$, however, was not statistically significant likely due to low number of events [12]. Future studies should assess the role of primary and secondary prophylaxis for ATE, especially in highrisk patients.

Rajshekhar Chakraborty ${ }^{1}$, Lisa Rybicki ${ }^{2}$, Jason Valent ${ }^{3}$, Alex V. Mejia Garcia ${ }^{3}$, Beth M. Faiman ${ }^{3}$, Jack Khouri ${ }^{3}$ Christy J. Samaras ${ }^{3}$, Faiz Anwer (iD) ${ }^{3}$ and Alok A. Khorana (D) ${ }^{3 \times-1}$

${ }^{1}$ Multiple Myeloma and Amyloidosis Program, Herbert Irving Comprehensive Cancer Center, Columbia University Medical Center, New York, NY, USA. ${ }^{2}$ Blood and Marrow Transplant Program, Taussig Cancer Institute, Cleveland Clinic, Cleveland, $\mathrm{OH}$, USA. ${ }^{3}$ Department of Hematology and Medical Oncology, Taussig Cancer Institute, Cleveland Clinic, Cleveland, OH, USA. ${ }^{\bowtie}$ email: khorana@ccf.org

\section{REFERENCES}

1. Kristinsson SY, Pfeiffer RM, Björkholm M, Goldin LR, Schulman S, Blimark $C$, et al. Arterial and venous thrombosis in monoclonal gammopathy of undetermined significance and multiple myeloma: a population-based study. Blood. 2010;115:4991-8.

2. Libourel EJ, Sonneveld P, van der Holt B, de Maat MP, Leebeek FW. High incidence of arterial thrombosis in young patients treated for multiple myeloma: results of a prospective cohort study. Blood. 2010;116:22-26.

3. Kim SH, Lim KM, Noh JY, Kim K, Kang S, Chang YK, et al. Doxorubicin-induced platelet procoagulant activities: an important clue for chemotherapy-associated thrombosis. Toxicol Sci. 2011;124:215-24.

4. Bradbury CA, Craig Z, Cook G, Pawlyn C, Cairns DA, Hockaday A, et al. Thrombosis in patients with myeloma treated in the Myeloma IX and Myeloma XI phase 3 randomized controlled trials. Blood. 2020;136:1091-104.

5. Kristinsson SY, Pfeiffer RM, Björkholm M, Schulman S, Landgren O. Thrombosis is associated with inferior survival in multiple myeloma. Haematologica. 2012;97:1603-7.

6. Palumbo A, Rajkumar SV, Dimopoulos MA, Richardson PG, San Miguel J, Barlogie $B$, et al. Prevention of thalidomide- and lenalidomide-associated thrombosis in myeloma. Leukemia. 2008;22:414-23.

7. Maharaj S, Chang S, Seegobin K, Serrano-Santiago I, Zuberi L. Increased risk of arterial thromboembolic events with combination lenalidomide/dexamethasone therapy for multiple myeloma. Expert Rev Anticancer Ther. 2017;17:585-91.

8. Jaiswal $\mathrm{S}$ et al. Clonal Hematopoiesis and Risk of Atherosclerotic Cardiovascular Disease. 2017;377:111-21.

9. Mouhieddine TH, Sperling AS, Redd R, Park J, Leventhal M, Gibson CJ, et al. Clonal hematopoiesis is associated with adverse outcomes in multiple myeloma patients undergoing transplant. Nat Commun. 2020;11:2996.

10. Navi BB, Reiner AS, Kamel $H$, ladecola $C$, Okin PM, Elkind $M$, et al. Risk of arterial thromboembolism in patients with cancer. J Am Coll Cardiol. 2017;70:926-38.

11. Agnelli G, Gussoni G, Bianchini C, Verso M, Mandalà M, Cavanna L, et al. Nadroparin for the prevention of thromboembolic events in ambulatory patients with metastatic or locally advanced solid cancer receiving chemotherapy: a randomised, placebo-controlled, double-blind study. Lancet Oncol. 2009;10:943-9.

12. Khorana AA, McNamara MG, Kakkar AK, Streiff MB, Riess $H$, Vijapurkar U, et al. Assessing full benefit of rivaroxaban prophylaxis in high-risk ambulatory patients with cancer: thromboembolic events in the randomized CASSINI Trial. TH Open. 2020;4:e107-e112.

\section{AUTHOR CONTRIBUTIONS}

RC designed the research and wrote the paper. AAK designed the research and approved the final draft of the manuscript. LR performed statistical analysis, provided critical input in study design and approved the final draft of the manuscript. JV, AVMG, BMF, JK, CJS, and FA performed patient management, provided input in the paper, and approved the final draft of the manuscript.

\section{COMPETING INTERESTS}

The authors declare no competing interests.

\section{ADDITIONAL INFORMATION}

Supplementary information The online version contains supplementary material available at https://doi.org/10.1038/s41408-021-00513-4.

Correspondence and requests for materials should be addressed to A.A.K. 
Reprints and permission information is available at http://www.nature.com/ reprints

Publisher's note Springer Nature remains neutral with regard to jurisdictional claims in published maps and institutional affiliations.

(c) Open Access This article is licensed under a Creative Commons Attribution 4.0 International License, which permits use, sharing, adaptation, distribution and reproduction in any medium or format, as long as you give appropriate credit to the original author(s) and the source, provide a link to the Creative Commons license, and indicate if changes were made. The images or other third party material in this article are included in the article's Creative Commons license, unless indicated otherwise in a credit line to the material. If material is not included in the article's Creative Commons license and your intended use is not permitted by statutory regulation or exceeds the permitted use, you will need to obtain permission directly from the copyright holder. To view a copy of this license, visit http://creativecommons. org/licenses/by/4.0/.

(c) The Author(s) 2021 